\section{Cluster-Therapie sorgt für Compliance}

A nfangs noch kritisch beäugt, hat sich die Cluster-Immuntherapie als schnelle und gut verträgliche Alternative zu den konventionellen Applikationsschemata der spezifischen Hyposensibilisierung etabliert. Cluster-Schemata zeichnen sich durch eine besonders kurze Einleitungsphase aus und ermöglichen so das Erreichen der Erhaltungsdosis innerhalb kürzester Zeit, was die Compliance der Patienten fördert.

Mit Clustoid ${ }^{\circledR}$ bietet das Unternehmen Roxall das einzige Präparat an, das speziell für die Cluster-Immuntherapie entwickelt wurde. In Clustoid ${ }^{\circledR}$ kommen durch ein Hochpolymerisationsverfahren hergestellte Allergoide, so genannte Clustoide, zum Einsatz, die sowohl durch ihre Wirksamkeit als auch durch ihre Verträglichkeit überzeugen. Die Patienten werden innerhalb einer Woche mit nur vier Injektionen auf die Erhaltungsdosis gebracht. Diese schnelle Dosissteigerung ermöglicht einen Beginn der Immuntherapie noch knapp zwei Wochen vor Beginn der Pollensaison und stellt somit eine interessante Alternative dar für Patienten, die eine zeitaufwendige herkömmliche Therapie nicht durchführen lassen wollen.

Daten zur Verträglichkeit und zur Compliance bei Anwendung der Cluster-Immuntherapie mit Clustoid ${ }^{\circledR}$ wurden auf dem 4. Gemeinsamen Deutschen Allergiekongress im September in Berlin vorgestellt. In einer Prä-Post-Analyse wurden Patientenbefinden, Symptomatik und Medikamentenverbrauch bei 239 Patienten mit allergischer Rhinokonjunktivitis evaluiert. Die Ergebnisse nach einem Behandlungszeitraum von einem Jahr zeigten, dass die ClusterImmuntherapie mit Clustoid ${ }^{\circledR}$ eine sichere Therapieform ist, die eine signifikante Reduktion der allergischen Symptomatik und des Medikamentenbedarfs bewirkt: $98 \%$ der verabreichten 2.564 Injektionen wurden gut vertragen, schwere unerwünschte Reaktionen blieben aus. Weiter zeigte sich eine signifikante Verbesserung der Symptomatik
Rechtssicherheit in der Immuntherapie

Die im November 2008 in Kraft getretene Therapieallergene-Verordnung (TAV) bedeutet Rechtssicherheit für Patienten, Ärzte und Hersteller. Die damit eingeführte Chargenzulassung trägt dazu bei, dass alle Hersteller die gleichen Voraussetzungen zum Inverkehrbringen ihrer Präparate zur spezifischen Immuntherapie erbringen müssen. Für die Immuntherapeutika Clustoid $^{\circledR}$, Roxoid $^{\circledR}$ und Sulgen $^{\circledR}$ Spray wurden sämtliche Chargen gemäß TAV durch das Paul-Ehrlich-Institut (PEI) freigegeben und Neuzulassungen angemeldet, die den gehobenen internationalen Anforderungen des PEI und der europäischen Zulassungsbehörde EMEA entsprechen.

um durchschnittlich 60\%. Insgesamt berichteten $85 \%$ der Patienten nach einer einjährigen Behandlung mit Clusto$\mathrm{id}^{\circledR}$ von einer deutlichen Besserung ihres Befindens.

Nach Informationen von Roxall, Hamburg

\title{
Neue Ära in der Sublingualtherapie
}

$\mathrm{D}$ ie sublinguale Immuntherapie (SLIT) kann heute angesichts vieler neuer Studien, vor allem mit Allergentabletten, evidenzbasiert durchgeführt werden. Dies spiegelt sich auch in der neuen Leitlinie zur spezifischen Immuntherapie wider, in der SLIT und subkutane Hyposensibilisierung praktisch gleichberechtigt empfohlen werden. „Damit hat eine neue Ära in der spezifischen Immuntherapie begonnen", sagte Prof. Dr. Claus Bachert, Gent, Belgien. Bei der Therapieentscheidung sollte

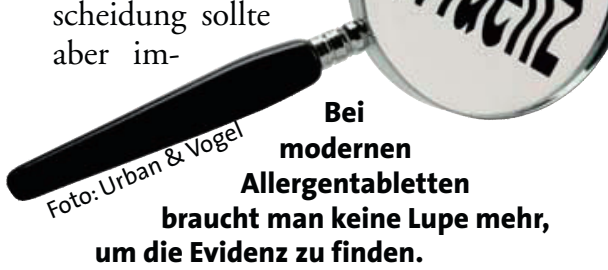

mer auch die Studienlage zu den einzelnen Allergenpräparaten berücksichtigt werden - eine wichtige Forderung, der auch vonseiten des Gesetzgebers jetzt mit der Therapieallergene-Verordnung Rechnung getragen wurde.

Eine klinische Wirksamkeit der SLIT nach den Kriterien der evidenzbasierten Medizin ist vor allem aus den Ergebnissen neuerer Studien ableitbar, die randomisiert, doppelblind und plazebokontrolliert durchgeführt wurden. Wichtig für die Belastbarkeit der Studienergebnisse sei auch eine ausreichende "Power“, erklärte Prof. Dr. Margitta Worm, Berlin. Nur mit einer ausreichend großen Patientenzahl lasse sich eine statistisch abgesicherte Wirksamkeit des Verums gegenüber der Plazebokontrolle darstellen. Erforderlich sind je nach Fragestellung 250 bis 260 Patienten. In Dosisfindungs- oder Zulas- sungsstudien, wie sie z.B. für die 5-Gräser-Tablette Oralair ${ }^{\circledR}$ oder die neue noch in Erprobung befindliche StallergenesMilbentablette durchgeführt wurden, sind die erforderlichen Patientenzahlen sogar noch deutlich größer.

Durch die umfassenden Zulassungsstudien für Oralair ${ }^{\circledR}$ werde dem Arzt eine wirksame und sichere Allergentherapie ermöglicht, erläuterte Priv.Doz. Dr. Randolf Brehler, Münster. Die therapeutisch optimale Erhaltungsdosis von 300 IR wurde in einer Dosisfindungsstudie ermittelt, belegt ist die Wirksamkeit im Rahmen eines prä- und cosaionalen Behandlungsschemas. $\quad b k$

Industriesymposium „Die spezifische Immuntherapie am Beginn einer neuen Ära - verändern Therapieallergene-Verordnung und die neuen Leitlinien die Therapieentscheidung?".4. Gemeinsamer Deutscher Allergiekongress, Berlin, 3.-6. September 2009. Veranstalter: Stallergenes, Kamp-Lintfort 